\section{TATRA \\ MDUNTaiNS \\ Mathematical Publications}

DOI: $10.2478 / \mathrm{tmmp}-2013-0007$

Tatra Mt. Math. Publ. 54 (2013), 83-92

\title{
STABILITY PROPERTIES OF A DISCRETIZED NEUTRAL DELAY DIFFERENTIAL EQUATION
}

\author{
JANA HrabaLOVÁ
}

\begin{abstract}
The paper discusses the asymptotic stability region of a discretization of a linear neutral delay differential equation

$$
x^{\prime}(t)=a x(t-\tau)+b x^{\prime}(t-\tau) .
$$

We present necessary and sufficient conditions specifying this region and describe some of its properties.
\end{abstract}

\section{Introduction and preliminaries}

In this paper, we discuss the asymptotic stability region of the Euler discretization applied to the test delay differential equation of the neutral type

$$
x^{\prime}(t)=a x(t-\tau)+b x^{\prime}(t-\tau), \quad t>0,
$$

where $a, b$ are real scalars and the delay $\tau$ is a positive real scalar. The problem of asymptotic stability of (11) is equivalent to a problem whether its corresponding characteristic equation

$$
\lambda-a \mathrm{e}^{-\lambda \tau}-b \lambda \mathrm{e}^{-\lambda \tau}=0
$$

has roots only with negative real parts and whether all its roots are uniformly negatively bounded away from the imaginary axis (see, e.g., [1] or [7]). This task has been studied in [4] for a more general neutral equation

$$
x^{\prime}(t)=a x(t-\tau)+b x^{\prime}(t-\tau)+c x(t)
$$

via analyzing the location of a pair of pure imaginary roots of its characteristic equation. Using the relevant result from [4] we may conclude that (1) is

(C) 2013 Mathematical Institute, Slovak Academy of Sciences.

2010 Mathematics Subject Classification: Primary 39A12, 34K20; Secondary 39A30.

Keywords: delay differential and difference equation, neutral type, asymptotic stability, the Euler method.

Supported by the grant P201/11/0768 of the Czech Science Foundation and by the project FSI-S-11-3 of Brno University of Technology. 


\section{JANA HRABALOVÁ}

asymptotically stable if and only if

$$
|b|<1, \quad-\left(1-b^{2}\right)^{1 / 2} \arccos b<a \tau<0 .
$$

These inequalities set up the asymptotic stability region of (11), i.e., the set of all real couples $(a, b)$ such that any solution of (11) is tending to zero as $t \rightarrow \infty$. We denote this region by $S_{\tau}^{*}$ and depict it on Figure 1.

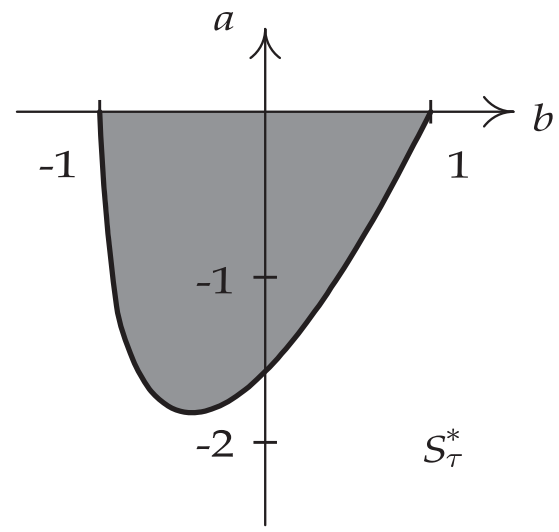

FIGURE 1. The asymptotic stability region $S_{\tau}^{*}$ for $\tau=1$.

The goal of this paper is to describe the asymptotic stability region for the basic (Euler) discretization of (1) and investigate some of its properties (for other related qualitative investigations of discretized delay differential equations we refer, e.g., to [8]).

The paper is organized as follows. In Section 2 we provide the forward Euler discretization of (11) and derive the necessary and sufficient conditions for its asymptotic stability. In Section 3 we discuss some properties of the derived asymptotic stability region and perform some comparisons with respect to $S_{\tau}^{*}$.

\section{The forward Euler discretization}

Let us consider a mesh $t_{n}=n h, n=0,1, \ldots$, where $h>0$ is a stepsize of the method. To avoid the interpolation in a replacement of delayed terms we impose $h=\tau / k, k \in \mathbb{Z}^{+}$. The forward Euler method for a delay differential equation

$$
x^{\prime}(t)=f(t, x(t), x(t-\tau))
$$

is a formula of the form

$$
y_{n+1}=y_{n}+h f\left(t_{n}, y_{n}, y_{n-k}\right), \quad n=0,1,2 \ldots,
$$


where $y_{n}$ is an approximate value of $x\left(t_{n}\right)$. The application of the forward Euler method to (1) yields a four-term linear difference equation

$$
\Delta y_{n}=b y_{n-k+1}+(a h-b) y_{n-k}, \quad n=0,1,2 \ldots,
$$

where $\Delta y_{n}=y_{n+1}-y_{n}$ is the forward difference operator.

In order to analyze its asymptotic stability we utilize the explicit necessary and sufficient conditions for a more general four-term linear difference equation

$$
y_{n+1}+\alpha y_{n}+\beta y_{n-k+1}+\gamma y_{n-k}=0, \quad n=0,1,2 \ldots,
$$

which have been recently derived in [2]. For other approaches in determining asymptotic stability of (3) we refer to [5] or [9].

We recall that (3) is asymptotically stable if $\lim _{n \rightarrow \infty} y_{n}=0$ for any solution of (3). It is well known that asymptotic stability of this linear difference equation depends on the location of the roots of its characteristic polynomial with respect to the unit disk (see, e.g., [3]). If the characteristic polynomial associated to (3)

$$
\lambda^{k+1}+\alpha \lambda^{k}+\beta \lambda+\gamma=0
$$

is of the Shur type, i.e., all its roots are located inside the open unit disk, then (3) is asymptotically stable (and conversely).

The following proposition, which is due to [2], gives the necessary and sufficient conditions guaranteeing that all the roots of (4) lie within the open unit disk.

Proposition 2.1. Let $\alpha, \beta$ and $\gamma$ be real constants and $k$ be a positive integer. Then all the roots of (4) lie inside the unit disk if and only if one of the following conditions holds:

(C1) $1+\alpha+\beta+\gamma>0, \quad 1+\alpha-\beta-\gamma>0, \quad 1-\alpha+\beta-\gamma>0, \quad 1-\alpha-\beta+\gamma>0$, and $k$ is any positive integer.

(C2) $1+\alpha+\beta+\gamma>0, \quad 1+\alpha-\beta-\gamma=0, \quad 1-\alpha+\beta-\gamma>0, \quad 1-\alpha-\beta+\gamma>0$, and $k$ is any positive integer.

(C3) $1+\alpha+\beta+\gamma>0 \quad 1+\alpha-\beta-\gamma>0, \quad 1-\alpha+\beta-\gamma=0, \quad 1-\alpha-\beta+\gamma>0$, and $k$ is any positive odd integer.

(C4) $1+\alpha+\beta+\gamma>0, \quad 1+\alpha-\beta-\gamma>0, \quad 1-\alpha+\beta-\gamma>0, \quad 1-\alpha-\beta+\gamma=0$, and $k$ is any positive even integer.

(C5) $1+\alpha+\beta+\gamma>0, \quad 1+\alpha-\beta-\gamma<0, \quad 1-\alpha+\beta-\gamma>0, \quad 1-\alpha-\beta+\gamma>0$, and $k$ is any positive integer such that

$$
k<\arccos \frac{\alpha^{2}-\beta^{2}+\gamma^{2}-1}{2|\alpha \gamma-\beta|} / \arccos \frac{\alpha^{2}-\beta^{2}-\gamma^{2}+1}{2|\alpha-\beta \gamma|} .
$$

(C6) $1+\alpha+\beta+\gamma>0, \quad 1+\alpha-\beta-\gamma>0, \quad 1-\alpha+\beta-\gamma<0, \quad 1-\alpha-\beta+\gamma>0$, and $k$ is any positive odd integer such that (5) holds.

(C7) $1+\alpha+\beta+\gamma>0, \quad 1+\alpha-\beta-\gamma>0, \quad 1-\alpha+\beta-\gamma>0, \quad 1-\alpha-\beta+\gamma<0$, and $k$ is any positive even integer such that (5) holds. 


\section{JANA HRABALOVÁ}

Let us note that (2) has a fixed parameter $\alpha=-1$ which allows us to simplify Proposition 2.1 in the following way.

CoRollary 2.2. Let $\beta$ and $\gamma$ be real constants and $k$ be a positive integer. Then all the roots of the polynomial

$$
\lambda^{k+1}-\lambda^{k}+\beta \lambda+\gamma=0
$$

lie inside the unit disk if and only if

$$
\beta+\gamma>0, \quad|\beta-\gamma|<2, \quad k<\arccos \frac{\gamma-\beta}{2} / \arccos \frac{2-\beta^{2}-\gamma^{2}}{2(1+\beta \gamma)} .
$$

Pr o of. Substituting $\alpha=-1$ into (C1)-(C7), the first two inequalities lead to a contradiction in all conditions except for (C5), where they become

$$
\beta+\gamma>0
$$

Further, the third and fourth inequalities of (C5) can be read as

$$
|\beta-\gamma|<2 \text {. }
$$

Setting $\alpha=-1$ in (5) we arrive at

$$
k<\arccos \frac{-\beta^{2}+\gamma^{2}}{2|\beta+\gamma|} / \arccos \frac{2-\beta^{2}-\gamma^{2}}{2|1+\beta \gamma|} .
$$

Since $\beta+\gamma>0$, the remaining issue is to determine the sign of $1+\beta \gamma$. Let us consider $\beta>0$ and $\gamma<0$. Then

$$
|\beta-\gamma|=\beta-\gamma<2,
$$

which implies

$$
1+\beta \gamma>1+\gamma(2+\gamma)=(1+\gamma)^{2} \geq 0
$$

The case $\beta<0$ and $\gamma>0$ is analogous. If $\beta$ and $\gamma$ are of the same sign, then the positivity of $1+\beta \gamma$ is obvious.

TheOREM 2.3. The discretization (2) is asymptotically stable if and only if

$$
a<0, \quad|a h-2 b|<2, \quad \tau \arccos \frac{2\left(1-b^{2}\right)+2 a b h-a^{2} h^{2}}{2\left(1+a b h-b^{2}\right)}<h \arccos \frac{2 b-a h}{2} .
$$

Pr o of. Theorem 2.3 is a direct consequence of the Corollary 2.2

Remark 2.4. The conditions of Theorem 2.3 define the asymptotic stability region of (2). We denote it by $S_{\tau}(h)$ to emphasise its dependence on the stepsize $h$. 


\section{STABILITY PROPERTIES OF DISCRETIZED NEUTRAL....}

\section{Some properties of $S_{\tau}^{*}$ and $S_{\tau}(h)$}

The aim of this section is to analyze mutual relationships between $S_{\tau}(h)$ with various $h$, as well as their comparisons with $S_{\tau}^{*}$.

This task has been studied in [6] for the equation (10) without a neutral term, that is,

$$
x^{\prime}(t)=a x(t-\tau), \quad t>0 .
$$

It was shown that the corresponding stability intervals $I_{\tau}(h)$, i.e., sets of all parameters $a$ such that any solution of the discretization (2) with $b=0$ tends to zero as $n \rightarrow \infty$, are enlarging with decreasing stepsize $h$. This corresponds to the well known behavior of the forward Euler discretization applied to (6) with $\tau=0$. Moreover, when $h$ is tending to zero, $I_{\tau}(h)$ is approaching the asymptotic stability interval of ([6). Here, we show that in the case of the neutral equation (1) the situation is more difficult.

TheOREM 3.1. Let $k_{1}<k_{2}$ be arbitrary positive integers and let $h_{1}=\tau / k_{1}>$ $\tau / k_{2}=h_{2}$ be corresponding stepsizes. Then there exist real couples $\left(a_{1}, b_{1}\right)$, $\left(a_{2}, b_{2}\right),\left(a_{3}, b_{3}\right)$ such that

(i) $\left(a_{1}, b_{1}\right) \notin S_{\tau}\left(h_{1}\right)$ and $\left(a_{1}, b_{1}\right) \in S_{\tau}\left(h_{2}\right)$,

(ii) $\left(a_{2}, b_{2}\right) \in S_{\tau}\left(h_{1}\right)$ and $\left(a_{2}, b_{2}\right) \in S_{\tau}\left(h_{2}\right)$,

(iii) $\left(a_{3}, b_{3}\right) \in S_{\tau}\left(h_{1}\right)$ and $\left(a_{3}, b_{3}\right) \notin S_{\tau}\left(h_{2}\right)$.

Pr o of. First, we consider $b=0$, i.e., (11) takes the form of (6). Via its discretization we get the difference equation

$$
y_{n+1}-y_{n}-a h y_{n-k}=0, \quad n=0,1,2 \ldots
$$

with the stability interval

$$
I_{\tau}(h)=\left\{a \in \mathbb{R}: 0>a>-\frac{2}{h} \sin \frac{\pi h}{4 \tau+2 h}\right\} .
$$

It was proved in [6] that the function defining the left endpoint of $I_{\tau}(h)$ is increasing which implies $I_{\tau}\left(h_{1}\right) \subset I_{\tau}\left(h_{2}\right) \subset I_{\tau}^{*}$. Moreover, both the inclusions are sharp. Let $\bar{a}$ be the left endpoint of $I_{\tau}\left(h_{1}\right)$, i.e.,

$$
\bar{a}=-\frac{2}{h_{1}} \sin \frac{\pi h_{1}}{4 \tau+2 h_{1}} .
$$

Then $\bar{a} \in I_{\tau}\left(h_{2}\right)$ and $\bar{a} \notin I_{\tau}\left(h_{1}\right)$. Consequently,

$$
(\bar{a}, 0) \notin S_{\tau}\left(h_{1}\right) \quad \text { and } \quad(\bar{a}, 0) \in S_{\tau}\left(h_{2}\right) .
$$

Thus, we have proved (i), where

$$
a_{1}=-\frac{2}{h_{1}} \sin \frac{\pi h_{1}}{4 \tau+2 h_{1}} \quad \text { and } \quad b_{1}=0 .
$$




\section{JANA HRABALOVÁ}

Setting $h=\tau$ we get

$$
I_{\tau}(\tau)=\left\{a \in \mathbb{R}: 0>a>-\frac{1}{\tau}\right\} .
$$

Because of the monotony property of $I_{\tau}(h)$, it holds

$$
(\tilde{a}, 0) \in S_{\tau}\left(h_{1}\right) \quad \text { and } \quad(\tilde{a}, 0) \in S_{\tau}\left(h_{2}\right) \text { for each } \tilde{a} \in I_{\tau}(\tau) \text {. }
$$

Consequently, it proves (ii) where $a_{2} \in(-1 / \tau, 0)$ and $b_{2}=0$.

Further, let us consider $b=-1$. The inequality

$$
\tau \arccos \frac{2\left(1-b^{2}\right)+2 a b h-a^{2} h^{2}}{2\left(1+a b h-b^{2}\right)}<h \arccos \frac{2 b-a h}{2}
$$

can be then simplify into

$$
a>-\frac{4}{h} \sin ^{2} \frac{\pi h}{2(\tau+h)} .
$$

We define

$$
f(h)=-\frac{4}{h} \sin ^{2} \frac{\pi h}{2(\tau+h)} .
$$

In order to analyze the monotony of $f(h)$, we drop the constraint $h=\tau / k$, $k \in \mathbb{Z}^{+}$and consider $f(h)$ to be a function of a continuous argument. Then

$$
f^{\prime}(h)=-\frac{2}{h}\left(-\frac{2}{h} \sin ^{2} \frac{\pi h}{2(\tau+h)}+\frac{\pi \tau}{(\tau+h)^{2}} \sin \frac{\pi h}{\tau+h}\right) .
$$

Obviously, $f^{\prime}(h)<0$ when

$$
-2 \sin ^{2} \frac{\pi h}{2(\tau+h)}+\frac{\pi \tau h}{(\tau+h)^{2}} \sin \frac{\pi h}{\tau+h}>0,
$$

or equivalently,

Let

$$
\tan \frac{\pi h}{2(\tau+h)}<\frac{\pi \tau h}{(\tau+h)^{2}}, \quad h>0
$$

$$
g_{1}(h)=\tan \frac{\pi h}{2(\tau+h)}, \quad g_{2}(h)=\frac{\pi \tau h}{(\tau+h)^{2}} .
$$

We show that $g_{1}(h)<g_{2}(h)$ for some $h>0$. Doing this, we investigate their derivatives

$$
g_{1}^{\prime}(h)=\frac{\pi \tau}{2(\tau+h)^{2}} \cos ^{-2} \frac{\pi h}{2(\tau+h)}, \quad g_{2}^{\prime}(h)=\frac{\pi \tau(\tau-h)}{(\tau+h)^{3}} .
$$

It holds

$$
g_{1}(0)=g_{2}(0) \quad \text { and } \quad g_{1}^{\prime}(h)<\frac{2 \pi \tau}{3(\tau+h)^{2}}, \quad h \in\left\langle 0, \frac{\tau}{2}\right) .
$$

It implies that

$$
g_{1}^{\prime}(h)<g_{2}^{\prime}(h) \quad \text { for all } h<\tau / 5 .
$$


Hence, $f(h)$ is decreasing for $h \in(0, \tau / 5)$. The remaining issue is to investigate the behavior of $f(h)$ for $h=\tau / \bar{k}, \bar{k}=1,2,3,4,5$. The values of $f(h)$ for such $h$ are computed in the following table.

TABle 1. The values of function $f(h)$ for some particular $h$.

\begin{tabular}{|c|c|c|c|c|c|}
\hline$h$ & $\tau$ & $\tau / 2$ & $\tau / 3$ & $\tau / 4$ & $\tau / 5$ \\
\hline$f(h)$ & $-2 / \tau$ & $-2 / \tau$ & $-1.757 / \tau$ & $-1.528 / \tau$ & $-1.400 / \tau$ \\
\hline
\end{tabular}

We may conclude that, with the exception of $h=\tau$ and $h=\tau / 2, f(h)$ is decreasing in $h=\tau / k, k \in \mathbb{Z}^{+}$. Therefore, by setting

$$
\hat{a}=-\frac{4}{h_{2}} \sin ^{2} \frac{\pi h_{2}}{2\left(\tau+h_{2}\right)}, \quad k_{2}=\frac{\tau}{h_{2}}>2
$$

we get $(\hat{a},-1) \in S_{\tau}\left(h_{1}\right)$ and $(\hat{a},-1) \notin S_{\tau}\left(h_{2}\right)$ for $k_{2}>2$.

To complete the proof we have to find a couple $(\check{a}, \check{b})$ such that $(\check{a}, \check{b}) \in S_{\tau}(\tau)$ and $(\check{a}, \breve{b}) \notin S_{\tau}(\tau / 2)$. To this purpose we investigate the stability boundary given by

$$
\tau \arccos \frac{2\left(1-b^{2}\right)+2 a b h-a^{2} h^{2}}{2\left(1+a b h-b^{2}\right)}=h \arccos \frac{2 b-a h}{2}
$$

in the neighborhood of $(-2 / \tau,-1)$, because this point is a common boundary point for both $S_{\tau}(\tau)$ and $S_{\tau}(\tau / 2)$. Using the implicit differentiation formula we get

$a^{\prime}(b)=\frac{\tau a(a h-2 b)+2\left(1-b^{2}\right)+2 a b h}{\tau\left[2\left(1-b^{2}\right)+a b h\right]+h\left(1+a b h-b^{2}\right)}, \quad 4-(2 b-a h)^{2} \neq 0,1+a b h-b^{2} \neq 0$.

Setting $h=\tau$ we obtain $a^{\prime}(-1)=1 / \tau$, while for $h=\tau / 2$ it holds $a^{\prime}(-1)=0$.

Hence, there exists a point $\check{b}$ in a left neighborhood of $b=-1$ such that $(-2 / \tau, \check{b}) \in S_{\tau}(\tau)$ and $(-2 / \tau, \check{b}) \notin S_{\tau}(\tau / 2)$. Thus, we have proved (iii), where for $k_{2}>2$,

$$
a_{3}=-\frac{4}{h_{2}} \sin ^{2} \frac{\pi h_{2}}{2\left(\tau+h_{2}\right)} \quad \text { and } \quad b_{3}=-1
$$

while for $k_{2}=2$, we have

$$
a_{3}=-2 / \tau \text { and } b_{3}=-1-\epsilon,
$$

where $\epsilon$ is a sufficiently small positive number.

Remark 3.2. In order to study $S_{\tau}(\tau)$ it is convenient to rewrite the last relation in Theorem 2.3 as follows. Employing the formula

$$
2 \arctan x=\arccos \frac{1-x^{2}}{1+x^{2}}=2 \operatorname{arccot} \frac{1}{x}, \quad x>0,
$$




\section{JANA HRABALOVÁ}

we can convert the left-hand side of the relation into

$$
2 \tau \arctan \left(\frac{a^{2} h^{2}}{(2(1-b)+a h)(2(1+b)-a h)}\right)^{1 / 2} .
$$

On the right-hand side, we apply the formula

$$
\arccos x=2 \arctan \frac{\sqrt{1-x^{2}}}{1+x}, \quad-1 \leq x \leq 1
$$

and we get

$$
2 h \arctan \left(\frac{2(1-b)+a h}{2(1+b)-a h}\right)^{1 / 2}
$$

Hence, we arrive at

$$
\tau \arctan \left(\frac{a^{2} h^{2}}{(2(1-b)+a h)(2(1+b)-a h)}\right)^{1 / 2}<h \arctan \left(\frac{2(1-b)+a h}{2(1+b)-a h}\right)^{1 / 2},
$$

which can be simplified for $h=\tau$ as

$$
a>\frac{b-1}{\tau} .
$$

Considering also the remaining conditions of Theorem 2.3 we get the following necessary and sufficient asymptotic stability condition describing $S_{\tau}(\tau)$

$$
b-1<a \tau<\min (0,2(1+b)) .
$$

The behaviour of $S_{1}(h)$ is illustrated in Figure 2. The depicted curves are the asymptotic stability boundaries for different values of the stepsize $h$. In all the cases is the stability area bounded from above by the b-axis.

Remark 3.3. In order to investigate the behavior of $S_{\tau}(h)$ as $h \rightarrow 0$, we rewrite the last inequality in Theorem 2.3 as

$$
\tau<h \arccos \frac{2 b-a h}{2} / \arccos \frac{2\left(1-b^{2}\right)+2 a b h-a^{2} h^{2}}{2\left(1+a b h-b^{2}\right)} .
$$

We are interested in the limit

$$
L=\lim _{h \rightarrow 0}\left(h \arccos \frac{2 b-a h}{2} / \arccos \frac{2\left(1-b^{2}\right)+2 a b h-a^{2} h^{2}}{2\left(1+a b h-b^{2}\right)}\right) .
$$

Using the L'Hospital rule we get

$$
L=\lim _{h \rightarrow 0}\left[\left(\arccos \frac{2 b-a h}{2}+\frac{a h}{\left(4-(2 b-a h)^{2}\right)^{1 / 2}}\right) / \frac{-a\left(2\left(1-b^{2}\right)+a b h\right)}{\left(4-(2 b-a h)^{2}\right)^{1 / 2}\left(1+a b h-b^{2}\right)}\right] .
$$

Hence,

$$
L=-\frac{\left(1-b^{2}\right)^{1 / 2}}{a} \arccos b .
$$




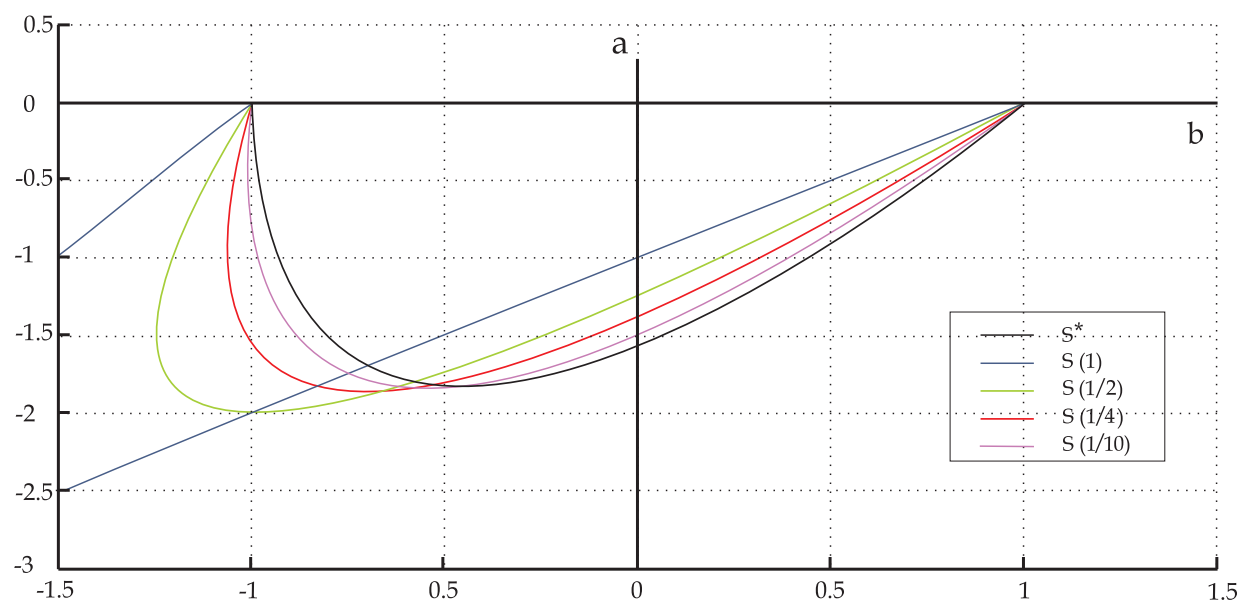

Figure 2. The asymptotic stability regions $S_{1}(h)$.

Therefore, (9) becomes

$$
a \tau>-\left(1-b^{2}\right)^{1 / 2} \arccos b,
$$

which, taking into account also the first two inequalities from Theorem 2.3 with $h=0$, is equivalent to the necessary and sufficient conditions on $S_{\tau}^{*}$.

Remark 3.4. Let $h=\tau / k$ where $k$ is an arbitrary positive integer. Then there exist real couples $\left(a_{1}, b_{1}\right),\left(a_{2}, b_{2}\right),\left(a_{3}, b_{3}\right)$ such that

(i) $\left(a_{1}, b_{1}\right) \notin S_{\tau}(h)$ and $\left(a_{1}, b_{1}\right) \in S_{\tau}^{*}$,

(ii) $\left(a_{2}, b_{2}\right) \in S_{\tau}(h)$ and $\left(a_{2}, b_{2}\right) \in S_{\tau}^{*}$,

(iii) $\left(a_{3}, b_{3}\right) \in S_{\tau}(h)$ and $\left(a_{3}, b_{3}\right) \notin S_{\tau}^{*}$.

The existence of $\left(a_{1}, b_{1}\right),\left(a_{2}, b_{2}\right)$ and $\left(a_{3}, b_{3}\right)$ can be proved using a similar approach as in Theorem 3.1 .

\section{REFERENCES}

[1] BELlen, A.-ZENNARO, M.: Numerical Methods for Delay Differential Equations, in: Numer. Math. Sci. Comput., Oxford University Press, Oxford, 2003.

[2] ČERMÁK, J.-JÁNSKÝ, J.-KUNDRÁT, P.: On necessary and sufficient conditions for the asymptotic stability of higher order linear difference equations, J. Difference Equ. Appl. 18 (2012), 1781-1800.

[3] ELAYDI, S.: An Introduction to Difference Equations. Springer, New York, 2005.

[4] FREEDMAN, H. I.-KUANG, Y.: Stability switches in linear scalar neutral delay equation, Funkcial. Ekvac. 34 (1991), 187-209. 


\section{JANA HRABALOVÁ}

[5] GUGLIELMI, N.: Delay dependent stability regions of $\Theta$-methods for delay differential equations, IMA J. Numer. Anal. 18 (1998), 399-418.

[6] HRABALOVÁ, J.: On stability intervals of Euler methods for a delay differential equation, in: Proc. of APLIMAT-11th Internat. Conf. on Appl. Math. (M. Kováčová, ed.), 2012, Bratislava, STU Bratislava, pp. 153-160.

[7] KOLMANOVSKII, V.-MYSHKIS, A.: Introduction to the Theory and Applications of Functional Differential Equations. Kluwer, Dordrecht, 1999.

[8] KUNDRÁT, P.: A note on asymptotic estimate for difference equation with several proportional delays, Tatra Mt. Math. Publ. 43 (2009), 109-114.

[9] LEVIN, S. A-MAY, R.: A note on difference delay equations, Theor. Popul. Biol. 9 (1976), 178-187.

Received October 12, 2012

Institute of Mathematics

Brno University of Technology

Technická 2

CZ-616-69 Brno

CZECH REPUBLIC

E-mail: yhraba05@stud.fme.vutbr.cz 